\title{
Dynamics of delayed-coupled chaotic logistic maps: influence of network topology, connectivity and delay times
}

 \\ ${ }^{1}$ Instituto de Física, Facultad de Ciencias, Universidad de la República, Iguá 4225, Montevideo 11400, Uruguay and \\ ${ }^{2}$ Departament de Fisica $i$ Enginyeria Nuclear, Universitat Politecnica \\ de Catalunya, Colom 11, E-08222 Terrassa, Barcelona, Spain
}

\begin{abstract}
We review our recent work on the synchronization of a network of delay-coupled maps, focusing on the interplay of the network topology and the delay times that take into account the finite velocity of propagation of interactions. We assume that the elements of the network are identical ( $N$ logistic maps in the regime where the individual maps, without coupling, evolve in a chaotic orbit) and that the coupling strengths are uniform throughout the network. We show that if the delay times are sufficiently heterogeneous, for adequate coupling strength the network synchronizes in a spatially homogeneous steady-state, which is unstable for the individual maps without coupling. This synchronization behavior is referred to as "suppression of chaos by random delays" and is in contrast with the synchronization when all the interaction delay times are homogeneous, because with homogeneous delays the network synchronizes in a state where the elements display in-phase time-periodic or chaotic oscillations. We analyze the influence of the network topology considering four different types of networks: two regular (a ring-type and a ring-type with a central node) and two random (free-scale Barabasi-Albert and small-world Newman-Watts). We find that when the delay times are sufficiently heterogeneous the synchronization behavior is largely independent of the network topology but depends on the networks connectivity, i.e., on the average number of neighbors per node.
\end{abstract}

PACS numbers: 05.45.Xt; 05.65.+b; 05.45.Ra

Keywords: Synchronization, coupled map lattices, time delays, logistic map

\section{INTRODUCTION}

A system composed of many nonlinear interacting units often forms a complex system with new emergent properties that are not held by the individual units. Such systems describe a wide variety of phenomena in biology, physics, and chemistry. The emergent property is usually synchronous oscillations. Examples include the synchronized activity in pacemaker heart cells, the cicardian rhythms, the flashing on-and-off in unison of populations of fireflies, synchronized oscillations in laser arrays, in Josephson junction arrays etc. [1, 2, 3, 4].

The effect of time-delayed interactions, which arise from a realistic consideration of finite communication times, is a key issue that has received considerable attention. The first systematic investigation of time-delayed coupling was done by Schuster and Wagner [5], who studied two coupled phase oscillators and found multistability of synchronized solutions. Since then, delayed interactions have been studied in the context of linear systems [6], phase oscillators [7], limit-cycle oscillators [8], coupled maps [9, 10], lasers [11, 12], neurons [13, 14, 15], etc.

It is well-known that oscillators that interact with different delay times can synchronize [16, 17, 18, 19, 20, 21], but the interplay of the interaction delays and the net-

\footnotetext{
*Electronic address: marti@fisica.edu.uy

${ }^{\dagger}$ Electronic address: mponce@fisica.edu.uy

${ }^{\ddagger}$ Electronic address: cristina.masoller@upc.edu
}

work topology is still poorly understood. Here we review the main results of our recent work on the synchronization of chaotic logistic maps [22, 23, 24] focusing on the interplay of delays and topology. We show that a network of delay-coupled logistic maps can synchronize, for adequate coupling strength, in spite of the fact that the interactions among the maps have an heterogeneous distribution of delay times. In the synchronized state the chaotic dynamics of the individual maps is suppressed and all elements of the network are in a steady-state, which is an unstable fixed-point of the uncoupled maps. This is in sharp contrast with the synchronized dynamics when the delays are homogeneous (instantaneous coupling and fixed-delay coupling), because with uniform delays the maps evolve in either periodic or chaotic orbits.

We investigate the influence of the network topology considering four different types of networks: two regular (a ring-type and a ring-type with a central node) and two random (free-scale Barabasi-Albert [26] and small-world Newman-Watts) 27]. We find that steady-state synchronization depends mainly on the average number of neighbors per node but is largely independent of the network architecture (i.e., the way the links are distributed among the nodes). This is also in contrast with the homogeneous delay case, because when the delay times are uniform the synchronization of the network depends strongly on the connection topology [10].

The rest of the paper is structured as follows. In Sec. III we introduce the basic ingredients of our model. Subsequently, Sec. III presents the results of the simulations. Finally, Sec. [IV summarizes the main findings and 
presents the conclusions from this investigation.

\section{NETWORKS AND INTERACTIONS MODEL}

We consider a network of $N$ coupled maps:

$$
x_{i}(t+1)=(1-\epsilon) f\left[x_{i}(t)\right]+\frac{\epsilon}{b_{i}} \sum_{j=1}^{N} \eta_{i j} f\left[x_{j}\left(t-\tau_{i j}\right)\right]
$$

where $t$ is a discrete time index, $i$ is a discrete spatial index $(i=1 \ldots N), f(x)=a x(1-x)$ is the logistic map, the matrix $\eta=\left(\eta_{i j}\right)$ defines the connectivity of the network: $\eta_{i j}=\eta_{j i}=1$ if there is a link between the $i$ th and $j$ th nodes, and zero otherwise. $\epsilon$ is the coupling strength, which is uniform throughout the network, and $\tau_{i j}$ is the delay time in the interaction between the $i$ th and $j$ th nodes (the delay times $\tau_{i j}$ and $\tau_{j i}$ need not be equal). The sum in Eq. (1) runs over the $b_{i}$ nodes which are coupled to the $i$ th node $\left(b_{i}=\sum_{j} \eta_{i j}\right)$. The normalized pre-factor $1 / b_{i}$ means that each map receives the same total input from its neighbors.

It can be noticed that the homogeneous steady-state

$$
x_{i}(t)=x_{j}(t)=x_{0}, \forall i, j, t,
$$

where $x_{0}$ is a fixed point of the uncoupled map, $x_{0}=$ $f\left(x_{0}\right)$, is a solution of Eq. (11) regardless of the delay times and of the connectivity of the network.

We will consider delay times that are either homogeneous $\left(\tau_{i j}=\tau_{0}\right.$ for all $\left.i, j\right)$ or heterogeneous. In the latter case we introduce a disorder parameter, $c$, that quantifies the degree of heterogeneity and allows varying the delays from a delta distribution (uniform delays) to Gaussian or exponential distributions. Specifically we consider

i) $\tau_{i j}=\tau_{0}+c \xi$, where $\xi$ is Gaussian distributed with zero mean and standard deviation one. The delays are homogeneous $\left(\tau_{i j}=\tau_{0}\right)$ for $c=0$ and are Gaussian distributed around $\tau_{0}$ for $c \neq 0$ [depending on $\tau_{0}$ and $c$ the distribution of delays has to be truncated to avoid negative delays, see Fig. 1(f)].

ii) $\tau_{i j}=\tau_{0}+c \xi$, where $\xi$ is exponentially distributed, positive, with unit mean. The delays are homogeneous $\left(\tau_{i j}=\tau_{0}\right)$ for $c=0$ and are exponentially distributed, decaying from $\tau_{0}$ for $c \neq 0$.

To investigate the influence of the topology we consider four networks, two of them are regular and the other two are random. The regular ones are a ring of nearest-neighbor elements (NN) while in the second one we added a central node connected to all other nodes (ST). The random networks consist of a scale free network (SF) constructed according to the Barabasi-Albert method 26] and, concerning the last one, we use the small-world (SW) topology proposed by Newman and Watts [27].

In the next section we present results of simulations that show that the homogeneous state-state, Eq. (2), with $x_{0}$ being the nontrivial fixed point of the logistic


FIG. 1: $x_{i}$ vs. $\epsilon$ (a) and (b); $\sigma^{2}$ vs. $\epsilon,(\mathrm{c})$ and (d). In Figs. 1 (a) and 1 (c) the delays are distributed exponentially $\left(\tau_{0}=0\right.$, $c=1.2$, see text); the distribution is shown in Fig. 1(e). In Figs. 1(b) and 1(d) the delays are Gaussian distributed $\left(\tau_{0}=3, c=2\right.$, see text); the distribution is shown in Fig. 1(f). The inset in Fig. 1(d) shows with detail the transition to synchronization: $\sigma^{2}$ decreases abruptly at $\epsilon \sim 0.4$, and is zero for $\epsilon>0.8$. $N=500, a=4$, and $p=0.3$.

map, $x_{0}=1-1 / a$, is a stable solution for adequate coupling strength and delay times.

\section{RESULTS}

The simulations were done choosing a random initial configuration, $x_{i}(0)$ randomly distributed in $[0,1]$, and letting the network evolve initially without coupling [in the time interval $\left.0<t<\max \left(\tau_{i j}\right)\right]$. We present results for $a=4$, corresponding to fully developed chaos of the individual maps, but similar results have been found for other values of the parameter $a$.

With both, either heterogeneous or homogeneous delay times, if the coupling strength is large enough the network synchronizes in a spatially homogeneous state: $x_{i}=x_{j} \forall i, j$. Figures 1 and 2 display the transition to synchronization as $\epsilon$ increases. In these figures the network has a small-world topology [27], but similar results have been found for other topologies, as discussed below. At each value of $\epsilon, 100$ iterates of an element of the network are plotted after transients. To do these bifurcation diagrams only the coupling strength, $\epsilon$, was varied; the network connectivity, $\eta_{i j}$, the delay times, $\tau_{i j}$, and the initial conditions, $x_{i}(0)$, are the same for all values of $\epsilon$.

Figure 1(a) displays results for exponentially distributed delays and Fig. 1(b) for Gaussian distributed delays; Fig. 2(a) for instantaneous interactions, and Fig. 2(b) for uniform delays. It can be noticed that for small $\epsilon$ the four bifurcation diagrams are very similar (we refer to this region as the "weak coupling region"); however, as $\epsilon$ increases above $\sim 0.1$ the bifurcation diagrams begin showing some differences, and for large $\epsilon$ they differ drastically: $x_{i}$ is constant in Figs. 1(a) and 1(b), $x_{i}=x_{0}=1-1 / a$, while $x_{i}$ varies within $[0,1]$ in Figs. 
(a)


FIG. 2: $x_{i}$ vs. $\epsilon$ (a) and (b); $\sigma^{2}$ vs. $\epsilon$ (c) and (d). In (a),(c) $\tau_{i j}=0 \forall i, j$; in (b),(d) $\tau_{i j}=3 \forall i, j$. All other parameters as in Fig. 1.

$2(\mathrm{a})$ and $2(\mathrm{~b})$.

To characterize the transition to synchronization we use the indicator

$$
\sigma^{2}=1 / N<\sum_{i}\left[x_{i}(t)-<x>\right]^{2}>_{t},
$$

where $\langle$.$\rangle denotes an average over the elements of the$ network and $\langle.\rangle_{t}$ denotes an average over time. Figures $1(\mathrm{c}), 1(\mathrm{~d}), 2(\mathrm{c})$ and $2(\mathrm{~d})$ display $\sigma^{2}$ vs. $\epsilon$ corresponding to the bifurcation diagrams discussed above. It can be noticed that for large $\epsilon$ there is in-phase synchronization in the four cases $\left[\sigma^{2}=0\right.$ if and only if $x_{i}(t)=x_{j}(t) \forall$ $i, j]$; however, we remark that an inspection of the timedependent dynamics reveals that the synchronized dynamics is different: for heterogeneous delays the maps are in a steady-state, while for homogeneous delays the maps evolve either periodically or chaotically. It can also be observed that the four plots of $\sigma^{2}$ vs. $\epsilon$ are similar in the weak coupling regime (in Fig. 2(d) the network synchronizes also in a window of small $\epsilon$; this occurs for odd delays as reported in [10]).

Next we analyze the influence of the network topology. Figure 3 displays a density plot of $\sigma^{2}$ as a function of the coupling strength, $\epsilon$, and the mean number of neighbors per node, $\langle b\rangle=\sum b_{i} / N$. The four panels correspond to the different networks mentioned above (SW, $\mathrm{SF}$, ST and NN); in these cases the Gaussian distribution of delays is the same in the four panels. Despite the differences when the number of neighbors is small, it can be observed that the synchronizability of the network is largely independent of the topology.

We also show in Fig. (4) for the sake of comparison two topologies (SW, NN) with a Gaussian delays distribution. Analyzing Fig. [4 it is possible to conjecture that it represents an intermediate behaviour between the Random Delay Gaussian distribution and the Fixed Delay interaction. Regarding the case of odd delays, it looks like to remain a trace of the "island of synchronization"
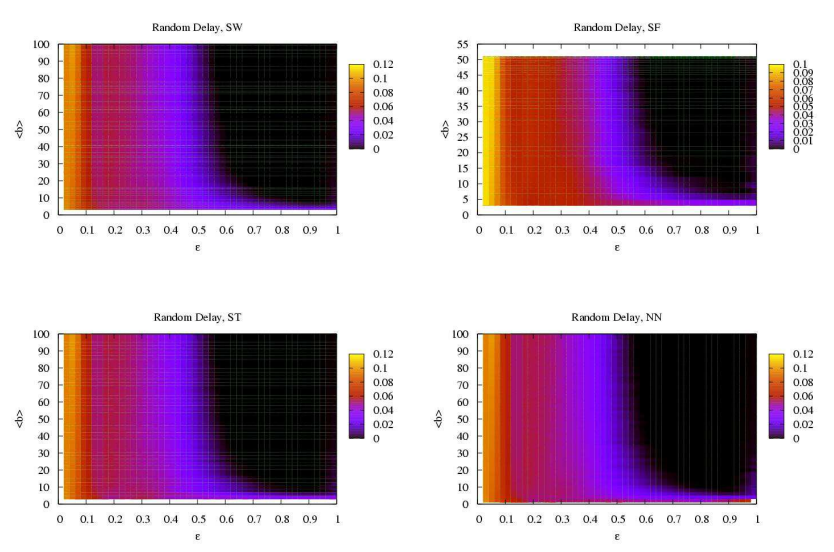

FIG. 3: (Color online) Random delays: synchronization regions for the four different networks considered and Gaussian distributed delays. The density plots represent the parameter $\sigma^{2}$ as a function of $\epsilon$ and $b$ averaged over 10 realizations of the initial conditions $(N=100$ and $a=4)$. Up: SW and SF, down: ST and NN.
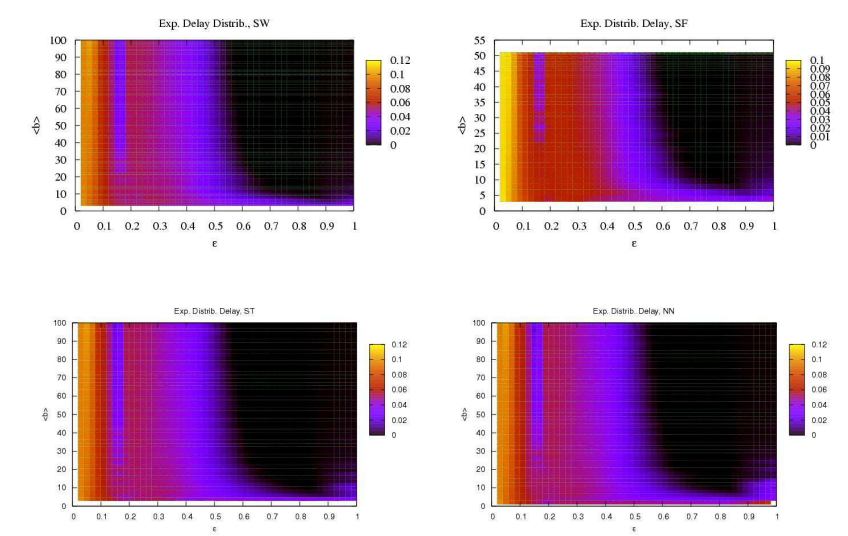

FIG. 4: (Color online) Random delays: synchronization regions for the SW, SF, ST and NN networks and exponential distributed delays. Same parameters as Fig. 3

present in the fixed delays interactions, but which is not present in the Gaussian Random Delay distribution.

Concerning the influence of the delay distribution, in Fig. [5] and Figs. 6 6 [7 we show the synchronization regions for the cases of instantaneous interactions and homogeneous delays respectively. It has been reported [10] that there is a very important difference between the case with an even or odd homogeneous delay (Figs. 66:7). With an even delay it can be prove that there is a kind of "island of synchronization", it is a region for $\epsilon$ (roughly speaking from $\epsilon \sim 0.15$ to 0.19 -weak coupling regime) where the maps synchronize. In addition to this, we see a subcritical period doubling bifurcation in this region. For an odd delay, this synchronization region disappears. Although this kind of delay, in the case of even 
delays, secure these "islands of synchronization" there are some particularities to take into account. For example, in the case of the NN topology, for small number of neighbors the synchronization is not as good as in the rest of the topologies. Also in the upper limit of the weak coupling regime $(\epsilon \gtrsim 0.4)$ there are differences between the irregular-networks (SW, SF) and the regular ones (NN, ST).
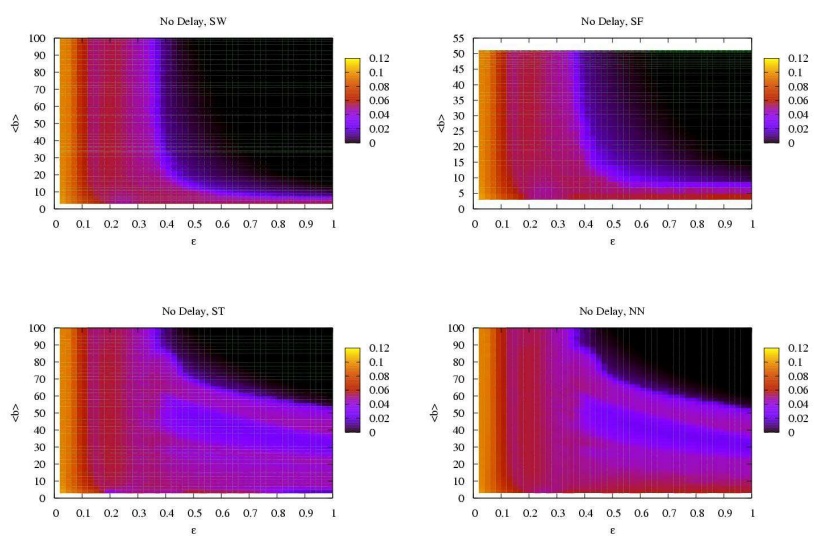

FIG. 5: (Color online) Instantaneous interactions: synchronization regions for the four different networks considered. Same parameters as Fig. 3 Up: SW and SF, down: ST and NN.

Considering all the showed delay distributions (Figs. 3145677) it is possible to conclude that the random delay distributions homogenizes the synchronization propensity in relation to the network topologies, and it also sets the value of the coupling constant $\epsilon$ for the on-set of synchronization in a greater $\epsilon$ compared with the other delay distributions. For instance, for random delays a "strong-coupling regime" starts roughly in


FIG. 6: (Color online) Homogeneous delays $\left(\tau_{0}=5\right.$ ): synchronization regions for the four different networks considered. Same parameters as Fig. 3 .
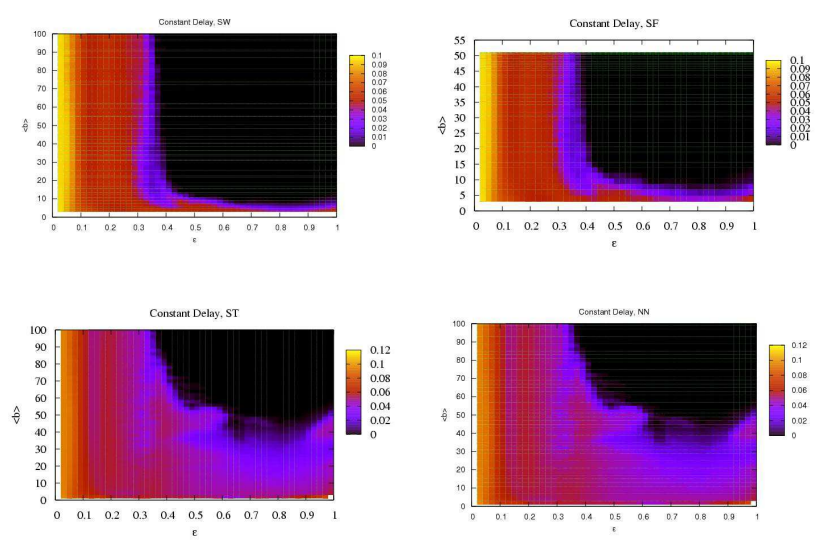

FIG. 7: (Color online) Homogeneous delays $\left(\tau_{0}=4\right)$ : synchronization regions for the SW (up-left), SF (up-right), ST (down-left) and NN (down-right) networks. Same parameters as Fig. 3 .

$\epsilon \sim 0.55$, while for homogeneous interactions this regime starts before $\epsilon \sim 0.4$, what is more for instantaneous interactions (no delay at all) it is not clear determined these region, i.e. because it depends on the topology and the average number of neighbours.

In further researches we plan to extend this investigation about the special weak-coupling regime and the properties of synchronization related to the network topology and connectivity. There we have analyzed the properties briefly discussed here, considering five different kind of topologies [29].

\section{DISCUSSION AND CONCLUSIONS}

To summarize, we studied the dynamics of a network of chaotic maps with delayed interactions and showed that the network synchronizes in a spatially homogeneous steady-state if the delay times are sufficiently heterogeneous. This behavior resembles the so-called "amplitude (or oscillator) death" phenomenon, which refers to the fact that under certain conditions the amplitude of delay-coupled oscillators shrinks to zero [8]. It has been shown that that distributed delays are more stabilizing than fixed delays [19]. The stabilization of the fixed-point is also related to the multiple delay feedback method proposed by Ahlborn and Parlitz [28], for stabilizing unstable steady states. We have recently compared the dynamics of a map of a network of $N$ delayed-coupled maps with the dynamics of a map with $N$ self-feedback delayed loops [23]. If $N$ is sufficiently large, we found that the dynamics of a map of the network is similar to the dynamics of a map with self-feedback loops with the same delay times. Several delayed loops stabilize the fixed point; however, the distribution of delays played a key role: if the delays are all odd a periodic orbit (and 
not the fixed point) was stabilized.

\section{Acknowledgments}

We acknowledge financial support from PEDECIBA and CSIC (Uruguay). A.C.M. thanks the Abdus Salam
ICTP for the hospitality and support on occasion of the workshop Perspectives on Nonlinear Dynamics 2007. CM acknowledges partial support from the European Commission (GABA project, FP6-NEST 043309).
[1] Y Kuramoto, Chemical Oscillations, Waves, and Turbulence (Springer-Verlag, Berlin, 1984).

[2] S Boccaletti, J Kurths, G Osipov, D L Valladares CS Zhou, Phys. Rep. 1, 366 (2002).

[3] A Pikovsky, M Rosenblum J Kurths Synchronization: A Universal Concept in Nonlinear Sciences (Cambridge University Press, Cambridge, England, 2003).

[4] S Manrubia, A S Mikhailov D H Zanette Emergence of Dynamical Order (World Scientific, Singapore, 2004).

[5] H G Schuster and P Wagner, Prog. Theo. Phys. 81, 939 (1987).

[6] V K Jirsa and M Ding, Phys. Rev. Lett. 93, 070602 (2004).

[7] E Niebur et al., Phys. Rev. Lett. 67, 2753 (1991); Ernst et al., Phys. Rev. Lett. 74, 1570 (1995); S Kim et al., Phys. Rev. Lett. 79, 2911 (1997); M K Stephen Yeung and S H Strogatz, Phys. Rev. Lett. 82, 648 (1999); M G Earl and S H Strogatz, Phys. Rev. E 67, 036204 (2003); M Denker et al., Phys. Rev. Lett. 92, 074103 (2004).

[8] D V Ramana Reddy et al., Phys. Rev. Lett. 80, 5109 (1998); R Herrero et al., Phys. Rev. Lett. 84, 5312 (2000).

[9] Y Jiang, Phys. Lett. A 267, 342 (2000); C Li et al., Physica A 335, 365 (2004).

[10] F M Atay et al., Phys. Rev. Lett. 92, 144101 (2004).

[11] J García-Ojalvo, J Casademont, C R Mirasso, M C Torrent, and J M Sancho, Int. J. Bifurcation Chaos 9, 2225 (1999).

[12] G Kozyreff, A G Vladimirov, and Paul Mandel, Phys. Rev. Lett. 85, 3809 (2000).

[13] J Foss, A Longtin, B Mensour, and J Milton, Phys. Rev. Lett.76, 708 (1996).
[14] A Takamatsu et al., Phys. Rev. Lett. 92, 228102 (2004); M Dhamala et al., Phys. Rev. Lett. 92, 074104 (2004).

[15] M Rosemblum and A Pikovsky, Phys. Rev. Lett. 92, 114102 (2004); Phys. Rev. E 70, 041904 (2004).

[16] K Otsuka and JL Chern, Phys. Rev. A 45, 5052 (1992).

[17] B Doiron et al., Phys. Rev. Lett. 93, 048101 (2004).

[18] D H Zanette, Phys. Rev. E. 62, 3167 (2000); S O Jeong et al., Phys. Rev. Lett. 89, 154104 (2002); T W Ko et al., Phys. Rev. E 69, 056106 (2004).

[19] F M Atay, Phys. Rev. Lett. 91, 094101 (2003).

[20] A C Martí and C Masoller, Phys. Rev. E 67, 056219 (2003); Physica A 342, 344 (2004).

[21] C M Gonzalez, C Masoller, M C Torrent and J GarcíaOjalvo, Europhys. Lett. 79, 64003 (2007).

[22] C Masoller A C Marti, Phys. Rev. Lett. 94, 134102 (2005).

[23] A C Martí, M. Ponce, and C. Masoller, Phys. Rev. E 72, 066217 (2005).

[24] A C Martí, M. Ponce C. and C. Masoller, Physica A 371, 104 (2006).

[25] S Boccaletti, V Latora, Y Moreno, M Chavez, D U Hwang, Phys. Rep. 424, 175 (2006).

[26] R Albert and A-L Barabási, Rev. Mod. Phys. 74, 47 (2002).

[27] M E J Newman and D J Watts, Phys. Rev. E 60, 7332 (1999); Phys. Letts. A 263, 341 (1999).

[28] A Ahlborn and U Parlitz, Phys. Rev. Lett. 93, 264101 (2004).

[29] A C Martí, M. Ponce C. and C. Masoller, in preparation (2007). 